\title{
Detection and characterization of small superficially located focal liver lesions by contrast-enhanced ultrasound with high frequency transducers
}

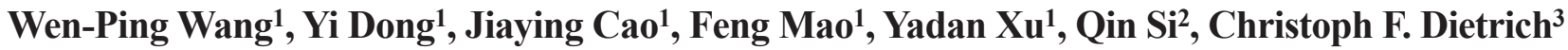

${ }^{1}$ Department of Ultrasound, Zhongshan Hospital, Fudan University, Shanghai, China, ${ }^{2}$ Department of Ultrasound,

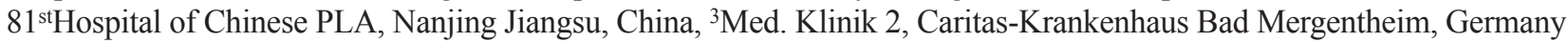

\begin{abstract}
Aim: To evaluate the benefits of contrast-enhanced ultrasound (CEUS) with high frequency transducers in the detection and characterization of small superficially located focal liver lesions (FLLs). Material and methods: From January 2015 to October 2016, 27 superficial FLLs detected by MRI were examined, first with the low frequency convex transducer (1-5 MHz) and afterwards with a high frequency transducer (7.5-12 MHz). High frequency dynamic CEUS was performed after a bolus injection of $2.4 \mathrm{ml}$ SonoVue ${ }^{\circledR}$. FLLs were confirmed by histopathology after surgery $(\mathrm{n}=18)$ or ultrasound guided 18 -gauge core-needle biopsy $(n=8)$, or by MRI follow-up $(n=1)$. Results: The mean diameter of FLLs was $10.5 \pm 3.1 \mathrm{~mm}$, and mean depth was $6.2 \pm 5.3 \mathrm{~mm}$. While using a high frequency transducerthe detection rate of FLLs $(100 \%, 27 / 27)$ was significantly higher than low frequency transducer $(25.9 \%, 7 / 27)(\mathrm{p}<0.05)$. The overall sensitivity, specificity and diagnostic accuracy were significantly improved by CEUS with a high frequency transducer (sensitivity $88.9 \%$; specificity $92.6 \%$; accuracy $96.2 \%$ ). Conclusion: Combining CEUS with the performance of high-frequency transducers is a promising technique to improve the detection and accurate diagnosis of small and superficial FLLs appearing indistinctive on BMUS.

Keywords: contrast-enhanced ultrasound (CEUS); haemangioma; metastasis; guidelines; B-mode ultrasound; hepatocellular carcinoma
\end{abstract}

\section{Introduction}

Early detection and accurate characterization of small focal liver lesions (FLLs) is crucial for an optimal curative treatment, which allows successful tumor surgery or radiofrequency ablation (RFA) with real-time ultrasound guidance [1-3].

Conventional ultrasound examination of liver is typically performed using a low frequency 2 to $5 \mathrm{MHz}$ transducer for adult patients [4,5]. Detection of small FLLs of

Received 18.08.2017 Accepted 17.09.2017

Med Ultrason

2017, Vol. 19, Nr. 4, 349-356

Corresponding author: Prof. Dr. med. Christoph F. Dietrich, MBA Internist, Hämato-Onkologe, Gastroenterologe und Proktologe, Pneumologe, Geriater/Arzt für Palliativmedizin

Chefarzt der Medizinischen Klinik 2

Uhlandstr. 7, 97980 Bad Mergentheim

Phone: 0793158 2201, Fax: 07931582290

E-mail: Christoph.dietrich@ckbm.de less than $5 \mathrm{~mm}$ always is challenging as these lesions often appear isoechoic on B mode ultrasound (BMUS); the same is true for superficial FLLs close to the liver capsule [6]. Over the last decade, with the wide clinical application of microbubble ultrasound contrast agents, contrastenhanced ultrasound (CEUS) has noticeably improved the identification and characterization of FLLs, especially for FLLs less than $10 \mathrm{~mm}$ in size [7-12]. However, it still proves to be difficult to differentiate malignant and benign FLLs, especially when the lesion is smaller than $10 \mathrm{~mm}$ or located superficially $[2,13]$.

Compared to the usual low frequency convex transducers (1-5 MHz), the utilization of high frequency 7 to $9 \mathrm{MHz}$ (or even higher) transducers offers important advantages such as high resolution and near field investigations. These transducers have been primarily used in transabdominal ultrasound examinations to improve the detection of liver surface nodularity [14-16] or for the detection of small FLLs as deep as 4 to $5 \mathrm{~cm}$ [17]. 
CEUS with high frequency transducers (HF-CEUS) is also becoming increasingly more available for the differentiation between benign and malignant FLLs $[6,18,19]$. Initial studies using dynamic CEUS with multifrequency matrix probes have indicated a high diagnostic value for the evaluation of tumor microvascularization in animal experiments $[19,20]$. Recent publications in intra-operative CEUS with high frequency transducers exhibited high diagnostic potential for the detection and differentiation between benign and malignant FLLs [2,11-13,21], particularly for liver lesions less than $10 \mathrm{~mm}$. The detection rate was comparable to pre-operative contrast enhanced spiral computer tomography (ceCT) and contrast enhanced magnetic resonance imaging (ceMRI) $[3,17]$. However, the value of HF-CEUS in regular transabdominal ultrasound examinations has not yet been evaluated.

The purpose of our current study was to evaluate the benefits of BMUS and CEUS with a high frequency transducer $(7.5-15 \mathrm{MHz})$ in identifying and characterization of small and superficial FLLs.

\section{Material and methods}

This prospective study was approved by our institutional review board. All patients gave their full informed consent before the CEUS examination. The procedure followed was in accordance with the Declaration of Helsinki.

\section{Patients}

From January 2015 to October 2016, 557 patients (138 females, 319 males) aged $21-87$ years with suspected FLLs were enrolled. Among them, 27 patients ( 8 females, 19 males) with 27 superficial FLLs were included in our current study. All those 27 FLLs were first suspected by MRI.The final diagnoses for 27 patients were based on histopathologic results by surgery $(\mathrm{n}=18)$ or ultrasound guided 18-gauge core-needle biopsy $(\mathrm{n}=8)$. Final diagnosis of one patient was established by MRI follow-up (Table I).

\section{Examination technique and image analysis}

Two experienced radiologists (more than 18 years' experience in CEUS of the liver), who were aware of the patients' clinical histories, performed ultrasound scanning with a Philips iU22 (Philips Bothell, WA, USA; C5-1 convex array transducer and L9-3 high frequency transducer), LOGIQ E9 (GE Healthcare, Milwaukee, WI, USA; C1-5 convex array transducer and L9-3 linear array high frequency transducer), or Aplio 500 (Toshiba Medical, Otawara, Japan, PVT375MV curved array transducer and linear PLT-1204 BT transducer).

\section{$B$ mode ultrasound with low frequency transducer (LF-BMUS)}

First, conventional transabdominal BMUS was performed with a low frequency convex array transducer
Table I. Baseline characteristics of patients

\begin{tabular}{ll}
\hline Characteristic & Patients $(\mathbf{n}=\mathbf{2 7})$ \\
\hline Age (year) & \\
$\quad$ Mean \pm SD & $51 \pm 12$ \\
$\quad$ Range & $28-77$ \\
Male/female & $19 / 8$ \\
Underlying liver diseases & \\
$\quad$ Cirrhosis & 11 \\
$\quad$ Fibrosis & 10 \\
$\quad$ Previous tumor history & 2 \\
$\quad$ None & 4 \\
AFP (ng/mL) & $16(59.3 \%)$ \\
$\quad \leq 20, \mathrm{n}(\%)$ & $8(29.6 \%)$ \\
$\quad 21-200, \mathrm{n}(\%)$ & $3(11.1 \%)$ \\
$\quad>200, \mathrm{n}(\%)$ & $3(8.5 \%)$ \\
CA $19-9(\mu /$ ml) & $24(91.5 \%)$ \\
$\quad \leq 4.9, \mathrm{n}(\%)$ & \\
$\quad>4.9, \mathrm{n}(\%)$ & 18 \\
Final diagnosis & 8 \\
$\quad$ Liver surgery & 1 \\
$\quad$ Core needle biopsy & MR images follow-up
\end{tabular}

AFP - alpha-fetoprotein; CA19-9 - carbohydrate antigen 19-9

(1-5 MHz), including grey scale and color flow imaging (CDFI) analysis. Low frequency B mode ultrasound (LF-BMUS) was used to examine the whole liver and to search for the suspected superficial FLL on MRI images. Optimized instrument settings, such as focal zones, dynamic range, depth and application of harmonic imaging were used to improve visualization.

Compared to the surrounding liver parenchyma, the BMUS imaging findingsincluded: maximum diameter of each lesion, echogenicity (hyperechoic, hypoechoic or isoechoic; homogeneous or heterogeneous), shape (regular or lobulated), and delineation (ill- or well-defined). Color flow imaging methods were used to detect blood flow signals inside lesions. In lesions with pulsed arterial blood flow, resistance index (RI) was measured.

BMUS and CEUS with high frequency transducer (HF-BMUS and HF-CEUS)

After low frequency ultrasound, high frequency B mode ultrasound (HF-BMUS) with a high frequency transducer (7.5-12 MHz) was carried out in areas with suspicious superficial FLLs. The same BMUS imaging criteria as for low frequency transducer was applied. The grey scale and CDFI imaging features were recorded and compared.

Then targeted HF-CEUS was performed injecting a bolus of $2.4 \mathrm{ml}$ SonoVue ${ }^{\circledR}$ (Bracco, Milan, Italy) intravenously followed by $5 \mathrm{ml}$ of normal sterile saline flush via a 22-gauge peripheral intravenous cannula. Low mechanical index (MI) ranging from 0.05 to 0.08 was used for 
real time CEUS imaging. Immediately after the injection of CEUS agents, 2 examiners evaluated the dynamic enhancement pattern of each lesion in comparison to the surrounding liver parenchyma. The CEUS enhancement of suspected focal liver lesions throughout the arterial, portal venous, and late phases (hypoenhancing, hyperenhancing, isoenhancing) and additional features of enhancement (e.g., rim-like or complete enhancement) were focused and observed according to the EFSUMB guideline $[4,5]$.

Each examination lasted at least 5 minutes after the bolus injection. Special attention was paid to the presence or absence of early arterial enhancement and to the detection of any portal venous or late phase (PVLP) washout area of contrast agents. In lesions with hypoenhancement during PVLP, a further $2.4 \mathrm{ml}$ bolus of SonoVue ${ }^{\circledR}$ was administered to improve visualization of lesion enhancement during the arterial phase, after an interval time of at least 15 minutes to allow for clearance of the previously injected contrast agents.

Digital cineloops were stored in a PC-based workstation connected to the ultrasound equipment. A 4-point scale was used to grade detection confidence of BMUS with low or high frequency transducer and CEUS with high frequency transducer: 1 , distinctive; 2 , probably visible; 3, poorly visible; 4, invisible [1].

\section{Statistical analysis}

Statistical analysis was performed with a computer software package (SPSS, version 21.0, IBM corporation, Armonk, USA). The improvement in diagnostic confidence was compared by McNemar analysis. Fisher's exact test was used to compare the 4-point scale grades of LF-BMUS, HF-BMUS and HF-CEUS in 27 FLLs. For all tests a $p$ value $<0.05$ was considered statistically significant.

\section{Results}

\section{Final diagnosis of FLLs}

Single FLLs were detected in 16 patients and multiple lesions in 11 patients. For multiple FLLs, only the biggest one was evaluated in this study. The characteristics of the lesions are detailed in Table II.

\section{Detection rate of FLLS}

On LF-BMUS (1-5 MHz), most of FLLs (20/27, $74.1 \%$ ) were isoechoic with ill-defined margins. Using a high frequency transducer, all those FLLs were slightly hyperechoic $(n=13)$ or slightly hypoechoic $(n=14)$ heterogeneous lesions on HF-BMUS. In 7 cases with one suspicious lesion detected by conventional low frequency transducer, all of them could be detected by HF-BMUS. In 20 of the 27 patients $(74.1 \%)$, no definite lesion was detected with the $3.5 \mathrm{MHz}$ transducer, 15 (55.6\%) sus-
Table II. Baseline characteristics of focal liver lesions included in the study

\begin{tabular}{ll}
\hline Characteristic & Focal liver lesions (n= 27) \\
\hline Diameter $(\mathrm{mm})$ & $10.5 \pm 3.1$ \\
Mean \pm SD & $5-17$ \\
Range & $7(25.9 \%)$ \\
Location & $20(74.1 \%)$ \\
Left lobe, $\mathrm{n}(\%)$ & \\
Right lobe, $\mathrm{n}(\%)$ & $13(55.6 \%)$ \\
Lesion depth $(\mathrm{mm})$ & $7(25.9 \%)$ \\
$\quad<5, \mathrm{n}(\%)$ & $7(25.9 \%)$ \\
$5-10$ & $17(62.9 \%)$ \\
$>10, \mathrm{n}(\%)$ & $5(18.5 \%)$ \\
Final diagnosis, $\mathrm{n}(\%)$ & $4(14.8 \%)$ \\
Primary HCC & $1(3.7 \%)$ \\
Recurrence HCC & \\
Metastasis & \\
Haemangioma &
\end{tabular}

$\mathrm{HCC}$ - hepatocellular carcinoma

Table III. Detection of small and superficial focal liver lesions using low frequency B mode ultrasound and contrast enhanced ultrasound with a high frequency transducer

\begin{tabular}{lll}
\hline Characteristic & $\begin{array}{l}\text { Low } \\
\text { frequency } \\
\text { transducer } \\
(\mathbf{1}-\mathbf{5} \mathbf{M H z})\end{array}$ & $\begin{array}{l}\text { High } \\
\text { frequency } \\
\text { transducer } \\
\mathbf{( 7 . 5}-\mathbf{1 2} \\
\text { MHz) }\end{array}$ \\
\hline $\begin{array}{lll}\text { Grey scale ultrasound, n (\%) } \\
\text { Isoechoic }\end{array}$ & $20(74.1 \%)$ & 0 \\
Slightly hyperechoic & $3(11.1 \%)$ & $13(48.1 \%)$ \\
Slightly hypoechoic & $4(14.8 \%)$ & $14(51.9 \%)$ \\
Color flow imaging, n $(\%)$ & & $20(74.1 \%)$ \\
pulsed arterial flow signals & $6(22.2 \%)$ & $0.65 \pm 0.13$ \\
RI & $0.50 \pm 0.11$ & $24(88.9 \%)$ \\
$\begin{array}{l}\text { Detected by HF-CEUS, n }(\%) \\
\text { Arterial hyperenhancement }\end{array}$ & $/$ & $19(70.4 \%)$ \\
PVLP hypoenhancement & $/$ &
\end{tabular}

FLL - focal liver lesion; LF-BMUS - low frequency B mode ultrasound; RI - resistance index; HF-CEUS - contrast enhanced ultrasound with high frequency transducer; PVLP - portal venous and late phase.

picious lesions were found by HF-BMUS as well. On HF-CEUS, 24 (88.9\%) lesions displayed arterial enhancement, while 19 (70.4\%) lesions showed hypoenhancement during PVLP (Table III).

Applying the 4-point scale classification, LF-BMUS classified 7 (25.9\%) of the 27 superficial FLLs diagnosed on MR imaging as poorly or probably visible lesions. HFBMUS detected an additional $15(55.5 \%)$ lesions $(\mathrm{p}<0.05)$. After injection of ultrasound contrast agents, the detection rate of FLLs $(100 \%, 27 / 27)$ by HF-CEUS was significantly higher than that of conventional BMUS with low frequency transducer $(25.9 \%, 7 / 27, \mathrm{p}<0.05)$ (Table IV). 
Table IV. Results of the 4-point scale grade of low frequency $B$ mode ultrasound and contrast enhanced ultrasound with high frequency transducer in 27 focal liver lesions

\begin{tabular}{llll}
\hline 4-point scale & LF-BMUS & HF-BMUS & HF-CEUS \\
\hline Grade 1, invisible & 20 & 5 & 0 \\
Grade 2, poorly visible & 4 & 3 & 0 \\
Grade 3, probably visible & 3 & 7 & 3 \\
Grade 4, distinctive & 0 & 12 & 24 \\
\hline
\end{tabular}

FLL - focal liver lesion; LF-BMUS - B mode ultrasound with low frequency transducer; HF-BMUS - B mode ultrasound with high frequency transducer; HF-CEUS - contrast enhanced ultrasound with high frequency transducer. $\mathrm{p}=0.008$ (Fisher's exact test)

\section{Special diagnostic BMUS features with high frequency transducer}

While using BMUS with high frequency transducer, peripheral hypoechoic halos were detected in 10 small HCC lesions, which were not visible by low frequency transducer.

On color flow imaging, pulsed color Doppler of arterial flow signals were detected in $20(74.1 \%)$ lesions by HF-BMUS, while only in $6(22.2 \%)$ lesions by LFBMUS.

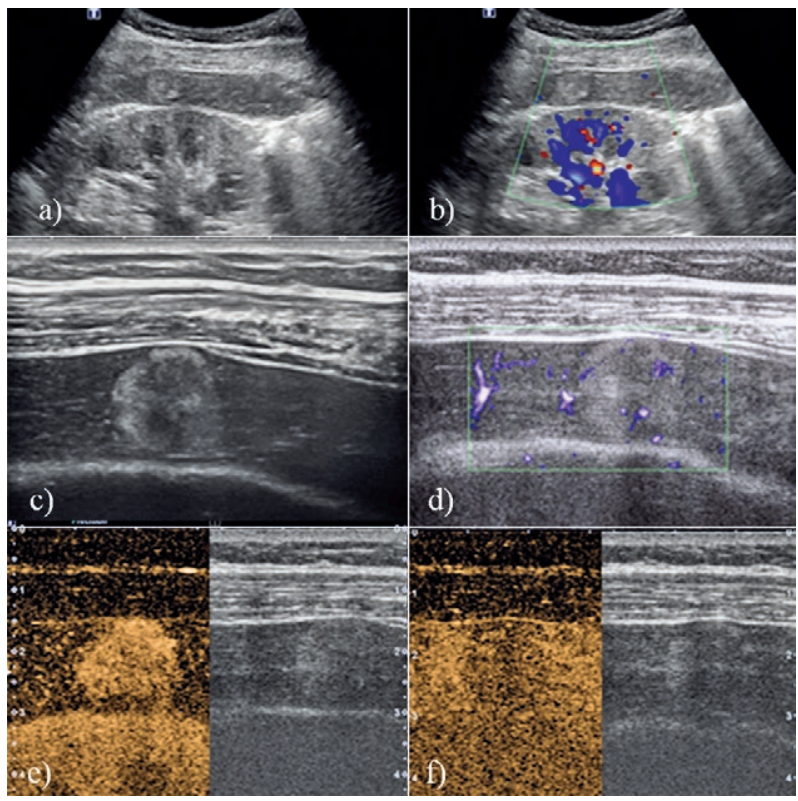

Fig 1. Small hepatocellular carcinoma (HCC) with $10 \mathrm{~mm}$ in diameter suspected by MRI was poorly visible on B mode ultrasound (BMUS) with low frequency transducer (a), no color flow signal could be detected inside the lesion (b). BMUS with high frequency transducer demonstrated a hyperechoic lesion with clear margin (c). Filiform pulsed color flow signals could be detected inside the lesion (d) with relatively high resistance index. After injection of contrast agents, the lesion was homogenously hyperenhancing during the arterial phase (e) and hypoenhancing during the portal venous and late phase (f). Finally, surgery confirmed a HCC.

\section{Contrast enhancement features of 27 FLLs with high frequency transducer}

On HF-CEUS, 16 (59.2\%) FLLs displayed rapid wash-in and rapid wash-out on CEUS, while 8 (29.6\%) lesions showed rapid or peripheral nodular wash-in and no wash-out, $3(11.1 \%)$ lesions showed isoenhancement and rapid wash-out.

After repeated injection of SonoVue ${ }^{\circledR}$, the contrast enhancement in the detected FLLs were evaluated during the arterial phase (10-30 seconds), portal venous (30-120 seconds) and late phases (120-300 seconds). On CEUS, homogeneous and complete hyperenhancement pattern during the arterial phase is highly suspicious for HCC in liver cirrhosis $(11 / 27,40.7 \%)$ (fig 1). Arterial rim-like hyper-enhancement and early wash out during PVLP is characteristic for metastatic malignancies $(3 / 27,11.1 \%)$. Arterial hyperenhancement and isoenhancement during PVLP is more common in recurrent HCCs $(3 / 27,11.1 \%$ ) (fig 2$)$. One haemangioma showed peripheral nodular and centripetal hyperenhancement during the arterial phase and isoenhancement during PVLP (fig 3) (Table V).

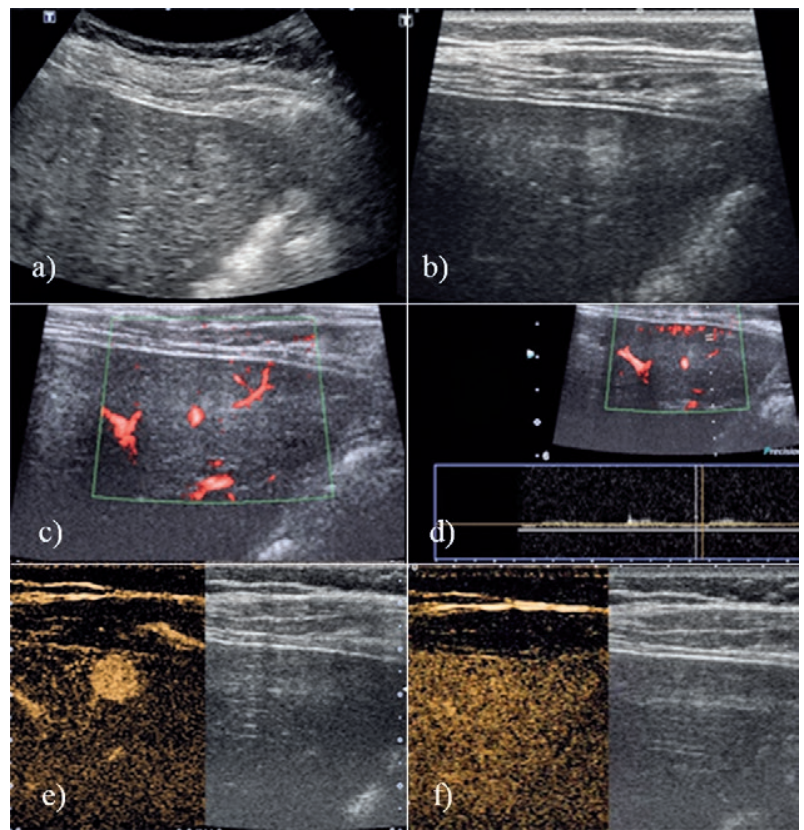

Fig 2. In a patient with known liver cirrhosis and previous resection of a HCC, a small lesion $(11 \mathrm{~mm})$ detected by MRI was invisible on B mode ultrasound (BMUS) with a low frequency transducer (a), BMUS with high frequency transducer demonstrated a slightly hyperechoic lesion (b) with pulsed arterial color flow signals (c) and high resistance index (d). On contrast enhancement with high frequency transducer, the lesion was homogenously hyperenhancing during the arterial phase (e) and isoenhancing during the portal venous and late phase (f). Finally, histology confirmed a recurrent HCC. 
Table V. Contrast enhancement features of 27 superficial focal liver lesions

\begin{tabular}{|c|c|c|c|c|}
\hline Characteristic & $\begin{array}{l}\text { Primary HCCs } \\
(\mathrm{n}=17)\end{array}$ & $\begin{array}{l}\text { Recurrence HCCs } \\
(\mathrm{n}=5)\end{array}$ & $\begin{array}{l}\text { Metastasis } \\
(n=4)\end{array}$ & $\begin{array}{l}\text { Haemangioma } \\
(\mathrm{n}=1)\end{array}$ \\
\hline \multicolumn{5}{|l|}{ Arterial phase enhancement, $\mathrm{n}(\%)$} \\
\hline Hyperenhanced & $16(94.1)$ & $3(60)$ & $4(100)$ & 1 \\
\hline Isoenhanced & $1(5.8)$ & $2(40)$ & 0 & 0 \\
\hline \multicolumn{5}{|l|}{ Type of arterial phase enhancement, $\mathrm{n}(\%)$} \\
\hline Diffuse homogeneous enhancement & $11(64.7)$ & $2(40)$ & 0 & 0 \\
\hline Diffuse inhomogeneous enhancement & $6(35.2)$ & $3(60)$ & $1(25)$ & 0 \\
\hline Rim-like hyperenhancement & 0 & 0 & $2(50)$ & 0 \\
\hline Nodular hyperenhancement & 0 & 0 & 0 & 1 \\
\hline \multicolumn{5}{|l|}{ PVLP enhancement, n (\%) } \\
\hline Hypoenhanced & $14(82.3)$ & $1(20)$ & $4(100)$ & 0 \\
\hline Isoenhanced & $3(17.7)$ & $4(80)$ & 0 & 1 \\
\hline
\end{tabular}

HCC - hepatocellular carcinoma; CEUS - contrast enhanced ultrasound.

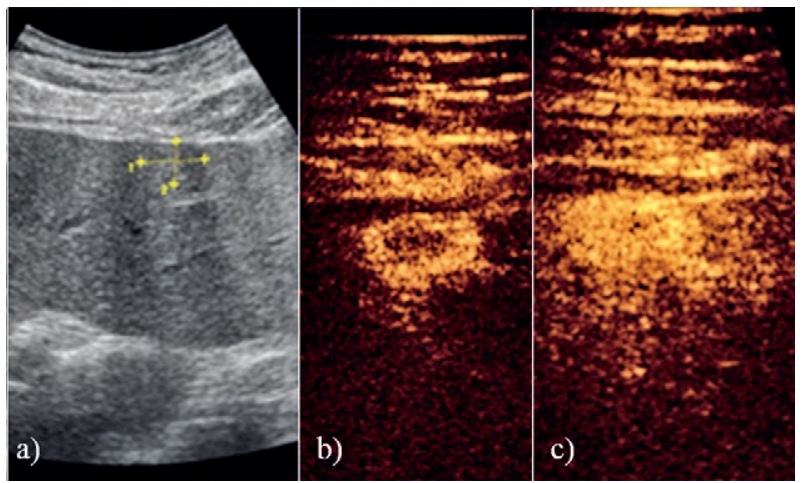

Fig 3. In a patient with previous history of a resected cystic renal cell carcinoma, a small and superficial lesion was suspected by B mode ultrasound (BMUS) with low frequency transducer (a). After the injection of contrast agents, the lesion showed peripheral nodular and centripetal enhancement during the arterial phase (b) and the portal venous and late phase(c). Finally, it was proved to be a hemangioma by the MRI follow-up.

The overall sensitivity, specificity and diagnostic accuracy for the correct characterization of malignant and benign small superficial FLLs were significantly improved by HF-CEUS compared to LF-BMUS (sensitivity: 88.9 vs $25.9 \%, \mathrm{p}<0.05$; specificity: 92.6 vs $18.5 \%, \mathrm{p}<0.05$; accuracy: 96.2 vs $18.5 \%, \mathrm{p}<0.01$, McNemar's test).

\section{Discussions}

Early detection and characterization of small FLLs may increase the chances of curative surgical resection or successful percutaneous ablation options of malignant tumours in early stages $[8,9,11,14,22,23]$. Conventional BMUS with low frequency convex transducer (1-5 MHz) is the first line imaging method for detecting and diagnosis of FLLs $[4,5]$. Assessing the liver surface nodularity using low frequency BMUS has specificity up to $95 \%$ [24] and a sensitivity of up to $91.1 \%$ indicative for cir- rhosis [25]. However, due to its limited resolution and various interfering factors or artefacts (such as liver cirrhosis background, intestinal gas interference, etc.) grey scale ultrasound or color flow Doppler techniques do not have the sensitivity to identify many of the vascular differences in FLLs. This technique fails to reliably differentiate between malignant and benign lesions [13,25-30]. Some FLLs are particularly difficult to diagnose even by biopsy because of their small size $(<10 \mathrm{~mm})$ or superficial location (close to liver capsule) $[12,15,26]$.

Recently, there has been a growing interest in the supplemental use of high-frequency transducers to better evaluate and delineate small FLLs near the surface in cirrhotic livers; these lesions are invisible using lowfrequency transducers $[15,24,25,31]$. Several studies have attempted to evaluate the use of high frequency transducers $(5-12 \mathrm{MHz})$ in the description of the liver surface and its implication for the presence of diffuse liver disease. Most of them focused on patients with liver cirrhosis $[15,16,25,32]$ or small hepatic metastasis from a known extrahepatic primary malignancy [14]. Jung et al described 9 cases in which small liver lesions (4-15 $\mathrm{mm}$ ) were detected during intraoperative ultrasound with high-frequency transducers. Some of these liver lesions had not been detected on computed tomography (CT) or MRI [19]. In another study, 38 patients with cirrhotic liver underwent an ultrasound examination using 3/3.5- 5 and $7.5 \mathrm{MHz}$ transducers to identify intraparenchymal regenerative nodules less than or equal to $20 \mathrm{~mm}$. Focal lesions were described in five patients. In only one case multiple lesions were revealed with the $7.5 \mathrm{MHz}$ probe, but not with any of the lower frequency transducers [32].

In our current study, all 27 FLLs cases diagnosed on MRI were in the superficial area of liver (mean lesion depth $6.2 \pm 5.3 \mathrm{~mm}$ ) and had a relatively small lesion size ranging from 5 to $17 \mathrm{~mm}$. LF-BMUS identified only 7 $(25.9 \%)$ lesions, classified as poorly or probably visible 
isoechoic lesions. Due to its high superficial temporal and spatial resolution, BMUS with high-frequency (7.5$12 \mathrm{MHz}$ ) transducer showed advantages in detection and localization of those superficial small FLLs. As shown in our results, HF-BMUS detected an additional 15 (55.5\%) lesions compared to LF-BMUS. Also, with HF-BMUS, we observed a clearer visualization of FLLs echogenicity, more distinct margins and more accurate measurement of lesions' size. Peripheral hypoechoic halos were detected in 10 small superficial HCC lesions, which were not visible using low frequency transducer. This sign might be very helpful for the diagnosis of liver malignancies $[11,13]$.

From the acoustic theory, BMUS with high frequency transducers is more sensitive in displaying nearfield blood flow signals than low frequency transducer $[16,19,32]$. In our results, more pulsed arterial color flow signals were detected inside the superficial liver lesions by HF-BMUS than by LF-BMUS ( $74.1 \%$ vs $22.2 \%)$. Investigation of intratumoral vessels can be helpful for further characterization of focal liver lesions, e.g. small HCC show detectable pulsed arterial flow signals caused by arteriovenous shunts $[11,21]$. This renders color Doppler flow imaging with a high frequency transducer a helpful technique to further characterize or differentiate superficial small FLLs.

In the last decade, CEUS has emerged as an important diagnostic tool and the physician's first diagnostic choice in many clinical situations $[13,21,33]$. According to the EFSUMB guideline, CEUS allows the characterization of most of FLLs by analysing the enhancement pattern during the arterial, portal venous and late phases $[2,4,5,13]$. Previous studies have shown that transabdominal CEUS with low frequency convex transducer is significantly better than unenhanced BMUS for the detection $[1,3]$ and classification $[7,10,33]$ of FLLs. For unclear small FLLs which could not be characterized with confidence by using transabdominal BMUS or Doppler ultrasound techniques, real-time CEUS has demonstrated the ability to characterize them reliably $[1,12,21]$.

High resolution linear probes suitable for abdominal CEUS have been developed lately. A recent preclinical mouse model experiment showed that CEUS using high frequency transducers could accurately depict the contrast perfusion characteristics of blood vessels in liver lesions and characterize the fine blood perfusion of tumors before metastatic nodules can be detected using conventional ultrasound techniques [34]. Using HF-CEUS, solid liver lesions between 2 and $11 \mathrm{~mm}$ (mean $3.5 \mathrm{~mm}$ ) size were detectable in a humanized tumor mouse model [34]. In addition, HF-CEUS is able to visualize capillary microcirculation of benign and malignant liver lesions smaller than $10 \mathrm{~mm}[6]$.
Our results indicate that transabdominal CEUS with high frequency transducers (7.5-12 MHz) could be used to detect small FLLs with a medium size of $10 \mathrm{~mm}$. Furthermore, the high spatial resolution allows real-time identification and evaluation of those small and superficial FLLs, which cannot be performed with conventional imaging [35]. After repeated injection of SonoVue ${ }^{\circledR}$, the dynamic recording of contrast enhancements of FLLs during the arterial phase, the portal venous phase and late phase was achieved and evaluated with the 7.5-12 $\mathrm{MHz}$ probe on the superficial areas of liver. Using HFCEUS, various benign and malignant FLLs showed the similar enhancement pattern known from LF-CEUS according to the current guidelines $[4,5]$. The sensitivity, specificity and overall diagnostic accuracy for the correct classification of malignant and benign FLLs were significantly higher using HF-CEUS compared to LF-BMUS. HF-CEUS is advantageous in establishing early detection and diagnosis of small and superficial FLLs, and helps to decide treatment strategies in these early stages [36,37]. In addition, for those patients who will undergo percutaneous ablation treatments, HF-CEUS might also be a valuable non-invasive diagnostic clinical option for monitoring treatment responses [35,38-40].

\section{Limitation of the study}

In our study, both BMUS and CEUS with high-frequency transducers were superior in detecting and characterizing superficial and small FLLs compared to low frequency ultrasound methods. On the other hand, high frequency transducers have their limitations in evaluating deeper structures due to their limited penetration depth. Although offering a useful adjunct, transabdominal ultrasound examinations with high frequency transducers cannot substitute scanning with a low frequency transducers. We also refer to the use of contrast enhanced endoscopic ultrasound in the detection of superficially located liver tumours $[38,41-43]$.

\section{Conclusion}

In conclusion, transabdominal BMUS and CEUS with adjunctive use of high-frequency transducers is a valid and convenient method which could provide added diagnostic values in establishing early detection and accurate diagnosis of small and superficial FLLs that are invisible or appear indistinctive on LF-BMUS.

\section{Acknowledgments}

This study was supported by the National Natural Science Foundation of China (NSFC Grant 81371577, NSFC Grant 81571676 and NSFC Grant 81501471). 


\section{Conflict of interest: none}

\section{References}

1. Dong Y, Wang WP, Gan YH, Huang BJ, Ding H. Radiofrequency ablation guided by contrast-enhanced ultrasound for hepatic malignancies: preliminary results. Clin Radiol 2014;69:1129-1135.

2. Dietrich CF, Cui XW, Boozari B, Hocke M, Ignee A. Contrast-enhanced ultrasound (CEUS) in the diagnostic algorithm of hepatocellular and cholangiocellular carcinoma, comments on the AASLD guidelines. Ultraschall Med 2012;33 Suppl 1:S57-S66.

3. Dietrich CF, Kratzer W, Strobe D, et al. Assessment of metastatic liver disease in patients with primary extrahepatic tumors by contrast-enhanced sonography versus CT and MRI. World J Gastroenterol 2006;12:1699-1705.

4. Claudon M, Dietrich CF, Choi BI, et al. Guidelines and good clinical practice recommendations for Contrast Enhanced Ultrasound (CEUS) in the liver - update 2012: A WFUMB-EFSUMB initiative in cooperation with representatives of AFSUMB, AIUM, ASUM, FLAUS and ICUS. Ultrasound Med Biol 2013;39:187-210.

5. Claudon M, Dietrich CF, Choi BI, et al. Guidelines and good clinical practice recommendations for contrast enhanced ultrasound (CEUS) in the liver--update 2012: a WFUMBEFSUMB initiative in cooperation with representatives of AFSUMB, AIUM, ASUM, FLAUS and ICUS. Ultraschall Med 2013;34:11-29.

6. Wege AK, Schardt K, Schaefer S, Kroemer A, Brockhoff G, Jung EM. High resolution ultrasound including elastography and contrast-enhanced ultrasound (CEUS) for early detection and characterization of liver lesions in the humanized tumor mouse model. Clinical Hemorheol Microcirc 2012;52:93-106

7. Dong Y, Zhang XL, Mao F, Huang BJ, Si Q, Wang WP. Contrast-enhanced ultrasound features of histologically proven small $(</=20 \mathrm{~mm})$ liver metastases. Scand J Gastroenterol 2017;52:23-28.

8. Dong Y, Wang WP, Mao F, et al. Contrast enhanced ultrasound features of hepatic cystadenoma and hepatic cystadenocarcinoma. Scand J Gastroenterol 2017;52:365-372.

9. Dong Y, Wang WP, Cantisani V, et al. Contrast-enhanced ultrasound of histologically proven hepatic epithelioid hemangioendothelioma. World J Gastroenterol 2016;22:47414749.

10. Chiorean L, Cantisani V, Jenssen C, Sidhu PS, Baum U, Dietrich CF. Focal masses in a non-cirrhotic liver: The additional benefit of CEUS over baseline imaging. Eur J Radiol 2015;84:1636-1643.

11. Dietrich CF, Sharma M, Gibson RN, Schreiber-Dietrich D, Jenssen C. Fortuitously discovered liver lesions. World J Gastroenterol 2013;19:3173-3188.

12. Strobel D, Bernatik T, Blank W, et al. Diagnostic accuracy of CEUS in the differential diagnosis of small $(</=20 \mathrm{~mm})$ and subcentimetric $(</=10 \mathrm{~mm})$ focal liver lesions in com- parison with histology. Results of the DEGUM multicenter trial. Ultraschall Med 2011;32:593-597.

13. Dietrich CF. Liver tumor characterization--comments and illustrations regarding guidelines. Ultraschall Med 2012;33 Suppl 1:S22-S30

14. Schacherer D, Wrede C, Obermeier F, Schölmerich J, Schlottmann K, Klebl F. Comparison of low and high frequency transducers in the detection of liver metastases. Dig Liver Dis 2006;38:677-682.

15. Buadu A, Meyer MA. Small liver nodule detection with a high-frequency transducer in patients with chronic liver disease: report of 3 cases. J Ultrasound Med 2013; 32: 355-359.

16. Hann LE, Bach AM, Cramer LD, Siegel D, Yoo HH, Garcia R. Hepatic sonography: comparison of tissue harmonic and standard sonography techniques. AJR Am J Roentgenol 1999; 173:201-206.

17. Loss M, Schneider J, Uller W, et al. Intraoperative high resolution linear contrast enhanced ultrasound (IOUS) for detection of microvascularization of malignant liver lesions before surgery or radiofrequeny ablation. Clin Hemorheol Microcirc 2012;50:65-77.

18. Echtenacher B, Wege AK, Schardt K et al. High-Resolution Ultrasound Including Contrast-Enhanced Ultrasound (CEUS) for the Detection of Gas Formation during Aspergillus Fumigatus Infection in Mice. Ultraschall Med 2016;37:277-282.

19. Jung EM, Ross CJ, Rennert J, et al. Characterization of microvascularization of liver tumor lesions with high resolution linear ultrasound and contrast enhanced ultrasound (CEUS) during surgery: First results. Clin Hemorheol Microcirc 2010;46:89-99.

20. Wilson SR, Burns PN. Microbubble-enhanced US in body imaging: what role? Radiology 2010;257:24-39.

21. Dietrich CF, Maddalena ME, Cui XW, Schreiber-Dietrich D, Ignee A. Liver tumor characterization--review of the literature. Ultraschall Med 2012;33 Suppl 1:S3-S10.

22. Chiorean L, Cui XW, Tannapfel A, et al. Benign liver tumors in pediatric patients - Review with emphasis on imaging features. World J Gastroenterol 2015;21:8541-8561.

23. Schreiber-Dietrich DG, Leuschner I, Tannapfel A, et al. Primary liver tumours in childhood. Z Gastroenterol 2015;53:1267-1275.

24. Colli A, Fraquelli M, Andreoletti M, Marino B, Zuccoli E, Conte D. Severe liver fibrosis or cirrhosis: accuracy of US for detection--analysis of 300 cases. Radiology 2003;227:89-94.

25. Simonovsky V. The diagnosis of cirrhosis by high resolution ultrasound of the liver surface. Br J Radiol 1999;72:2934.

26. Dietrich CF, Ignee A, Hocke M, Schreiber-Dietrich D, Greis C. Pitfalls and artefacts using contrast enhanced ultrasound. Z Gastroenterol 2011;49:350-356.

27. Dietrich CF, Ignee A, Trojan J, Fellbaum C, Schuessler G. Improved characterisation of histologically proven liver tumours by contrast enhanced ultrasonography during the portal venous and specific late phase of SHU 508A. Gut 2004;53:401-405. 
28. Trojan J, Hammerstingl R, Engels K, Schneider AR, Zeuzem S, Dietrich CF. Contrast-enhanced ultrasound in the diagnosis of malignant mesenchymal liver tumors. J Clin Ultrasound 2010;38:227-231.

29. Dietrich CF, Schuessler G, Trojan J, Fellbaum C, Ignee A. Differentiation of focal nodular hyperplasia and hepatocellular adenoma by contrast-enhanced ultrasound. Br J Radiol 2005;78:704-707.

30. Dietrich CF, Mertens JC, Braden B, Schuessler G, Ott M, Ignee A. Contrast-enhanced ultrasound of histologically proven liver hemangiomas. Hepatology 2007;45:11391145.

31. Ladenheim JA, Luba DG, Yao F, Gregory PB, Jeffrey RB, Garcia G. Limitations of liver surface US in the diagnosis of cirrhosis. Radiology 1992;185:21-23.

32. Giorgio A, Francica G, de Stefano G, et al. Sonographic recognition of intraparenchymal regenerating nodules using high-frequency transducers in patients with cirrhosis. J Ultrasound Med 1991;10:355-359.

33. Seitz K, Strobel D, Bernatik T, et al. Contrast-Enhanced Ultrasound (CEUS) for the characterization of focal liver lesions - prospective comparison in clinical practice: CEUS vs. CT (DEGUM multicenter trial). Parts of this manuscript were presented at the Ultrasound Dreilandertreffen 2008, Davos. Ultraschall Med 2009;30:383-389.

34. Horie S, Chen R, Li L, Mori S, Kodama T. Contrast-enhanced high-frequency ultrasound imaging of early stage liver metastasis in a preclinical mouse model. Cancer Lett 2013;339:208-213.
35. Dietrich CF, Averkiou MA, Correas JM, Lassau N, Leen E, Piscaglia F. An EFSUMB introduction into Dynamic Contrast-Enhanced Ultrasound (DCE-US) for quantification of tumour perfusion. Ultraschall Med 2012;33:344-351.

36. Dietrich CF, Lorentzen T, Appelbaum L, et al. EFSUMB Guidelines on Interventional Ultrasound (INVUS), Part III - Abdominal Treatment Procedures (Long Version). Ultraschall Med 2016;37:E1-E32.

37. Dietrich CF, Lorentzen T, Appelbaum L, et al. EFSUMB Guidelines on Interventional Ultrasound (INVUS), Part III - Abdominal Treatment Procedures (Short Version). Ultraschall Med 2016;37:27-45.

38. Dietrich CF, Dong Y, Froehlich E, Hocke M. Dynamic contrast-enhanced endoscopic ultrasound: A quantification method. Endosc Ultrasound 2017;6:12-20.

39. Meloni MF, Galimberti S, Dietrich CF, et al. Microwave ablation of hepatic tumors with a third generation system: locoregional efficacy in a prospective cohort study with intermediate term follow-up. Z Gastroenterol 2016;54:541-547.

40. Dietrich CF, Cui XW, Chiorean L, Appelbaum L, Leen E, Ignee A. Local ablative procedures of the liver. Z Gastroenterol 2015;53:579-590.

41. Braden B, Jenssen C, D'Onofrio M, et al. B-mode and contrastenhancement characteristics of small nonincidental neuroendocrine pancreatic tumors. Endosc Ultrasound 2017;6:49-54.

42. Fusaroli P, Saftoiu A, Dietrich CF. Contrast-enhanced endoscopic ultrasound: Why do we need it? A foreword. Endosc Ultrasound 2016;5:349-350.

43. Ignee A, Atkinson NS, Schuessler G, Dietrich CF. Ultrasound contrast agents. Endosc Ultrasound 2016;5:355-362. 